\title{
Energy Efficient Optical Access and Metro Networks
}

\author{
Luca Valcarenghi*, Member, IEEE, Isabella Cerutti, and Piero Castoldi, Member, IEEE \\ Scuola Superiore Sant'Anna \\ via G. Moruzzi 1, 56124, Pisa, Italy \\ Tel: (+39) 050549 2138,Fax: (+39)0505492194,e-mail: luca.valcarenghi@sssup.it
}

\begin{abstract}
A common consensus is that the utilization of communication networks in place of human travel, if possible, is one of the means of reducing GreenHouse Gas (GHG) emissions. Therefore a more efficient energy utilization in communication networks would have the double effect of not only lowering the energy consumption of the Information and Communications Technology (ICT) sector itself but also to lower the carbon footprint of many human activities. The deployment of optical networks in place of electronic networks is foreseen as a means for reducing communications network energy consumption. However even the energy consumption of optical networks can be optimized. In this paper current initiatives in optimizing energy utilization in optical networks are surveyed. Then two use cases are presented. The first one shows that allowing Optical Network Units (ONU) to switch to sleep-mode (i.e., stand-by), through hardware and control protocol, sensibly reduces optical access network energy consumption. The second one shows that a careful combination of optical and electronic devices during the design and the deployment of metro networks can lead to an optimal energy consumption.
\end{abstract}

Keywords: energy efficiency, OPEX, optical access, optical metro, sleep mode.

\section{INTRODUCTION}

The greenhouse effect caused by Green House Gas (GHG) emissions (i.e., carbon dioxide $\left[\mathrm{CO}_{2}\right]$, methane $\left[\mathrm{CH}_{4}\right]$, nitrous oxide $\left[\mathrm{NO}_{2}\right]$, and ozone $\left[\mathrm{O}_{3}\right]$, Hydrofluorocarbons [HFCs], Perfluorocarbons [PFCs], and Sulphur hexafluoride $\left[\mathrm{SF}_{6}\right]$ ) is recognized as the main cause of global warming [1][2]. Human activities are heavily contributing to the GHG emissions either directly (e.g., the aviation industry burns large amounts of fossil fuel, and this burning process releases GHGs) or indirectly ( e.g., the ICT industry consumes electricity, part of which is produced by burning fossil fuel). The quantity of GHGs (reported, for simplicity, in tons of equivalent $\mathrm{CO}_{2}$--$\mathrm{CO}_{2 \mathrm{e}}$ ) produced directly or indirectly is defined as "carbon footprint" [3].

Information and Communications Technologies (ICT) take a dual role in this scenario. On the one hand, thanks to virtualization, information transmissions and teleconferencing, ICT avoid the need to transport documents and people and, thus, decrease GHG emissions caused by transportation [4][5][6]. On the other hand, the ICT carbon footprint due to indirect emissions is reported to be already comparable to that of aviation and it is estimated that $2-10 \%$ of the $\mathrm{CO}_{2}$ produced by human activity comes from ICT [4][7]. Such emissions are expected to raise steadily in the next years, due to the increase of users and increased utilization of the virtualization as a side effect. However these conflicting roles can lead to a beneficial effect if the decrease in energy consumption achieved by optimizing communications networks and the related byproducts (e.g., lowering the energy consumption required by the equipment cooling) will overcome the increase in energy consumed by the ICT sector due to its increased utilization.

Currently, estimates indicate that about $37 \%$ of the ICT carbon emission is due to the production of the energy necessary to operate (i.e., the OPerational Expenditures - OPEX) the telecom infrastructure and devices (i.e., the communications networks), especially in the metro/access [4][8][9][10]. Metro/access networks drain about $60 \%$ of the total energy utilized by communications networks. Today most of access network segment is based on energy-demanding technologies, such as xDSL and wireless access. However, even with the introduction of optical fibre in the access, the energy consumption is forecast to grow with the average access bit rate increase [10][11]. Furthermore, the energy per bit requested by Passive Optical Networks (PONs) Optical Network Units (ONUs) is at the top among the communications network devices [12].

Several governmental and non-governmental, national and international organizations, such as the United Nations Framework Convention on Climate Change (UNFCCC) (http://unfccc.int/2860.php), the Global Footprint Network (http://www.footprintnetwork.com/), the Climate Group (http://www.theclimategroup.org/), the WWF (http://www.worldwildlife.org/climate/climatesavers2.html), the United States Environmental Protection Agency (EPA) (http://www.epa.gov/), the Lawrence Berkeley National Laboratory (www.lbl.gov) in the USA, the European Environmental Agency (EEA) (http://www.eea.europa.eu/), the European Commission with the Strategic Energy $\quad$ Technology (SET) Plan (http://ec.europa.eu/energy/technology/set_plan/set_plan_en.htm), the Global Action Plan (http://www.globalactionplan.org.uk/index.aspx), the Carbon Trust (http://www.carbontrust.co.uk/default.ct) in Europe, the Energy Conservation Centre Japan (ECCJ) (http://www.asiaeec-col.eccj.or.jp/index.html), are investigating and promoting policies for monitoring and reducing GHG emissions.

In the ICT sector, standardization bodies started initiatives to improve the energy-efficiency of ICT technologies and protocols. It is relevant to mention the International Telecommunication Union 
Telecommunication Standardization Sector (ITU-T) that established the Study Group 5 on "Environment and Climate Change" for the period 2009-2012 (http://www.itu.int/ITU-T/studygroups/com05/index.asp). Similarly, the Institute of Electrical and Electronics Engineers (IEEE) started the IEEE P802.3az Energy Efficient Ethernet Task Force to develop energy-efficient Ethernet standards (http://www.ieee802.org/3/az/public/index.html). In addition, academic and corporate initiatives for finding energy-efficient solutions targeting the ICT sector are rising worldwide. CANARIE (http://www.canarie.ca), the Canadian advanced network organisation, launched a green IT pilot program to develop an ICT infrastructure that uses clean energy sources to sustain ICT. In the USA, the Energy Efficient Digital Networks (http://efficientnetworks.lbl.gov/enet.html) project at Lawrence Berkeley National Lab targets Ethernet networks and investigates technologies for adjusting the transmission rate and supporting the sleep mode in Ethernet data links. In Europe, the FP7-funded BONE network of excellence (www.ict-bone.eu) introduced a specific task on green networking (Joint Action 5 within Work Package 21) and is actively pursuing various research efforts on the topic. In Japan, the NTT group launched the NTT Group Ecology Program 21 within which the NTT Group Vision for Environmental Contribution targets the reduction of the electrical power required by customers' communication equipments (http://www.ntt.co.jp/csr_e/2006report/ecology/01.html). In addition, a study of the impact of ICT on $\mathrm{CO}_{2}$ emission in Japan in the short term (until 2010) and in the long term (until 2050) future is performed. The study accounts for data coming from other sectors, such as public transportation, and tries also to understand to which extent human will be willing to accept virtualization through ICT [6]. Other studies are investigating ways for reducing the energy consumption of current communications networks, both optical and electronic, by designing and managing devices and communications networks in an energy-efficient manner.

\section{ENERGY EFFICIENT OPTICAL NETWORKS}

This section briefly surveys the several studies on how optical networks can decrease communications network energy consumption and how to optimize the energy consumed by optical networks The objective of such studies aims at reducing the power consumption of the individual devices and/or the overall networks. The main solutions are based on support of sleep mode in devices or proper optimization of provisioned resources in the optical network. Such solutions are applied to and currently investigated in each network segment as depicted in Figure 1. Thus, these efforts are classified based on the network segment that they are addressing.

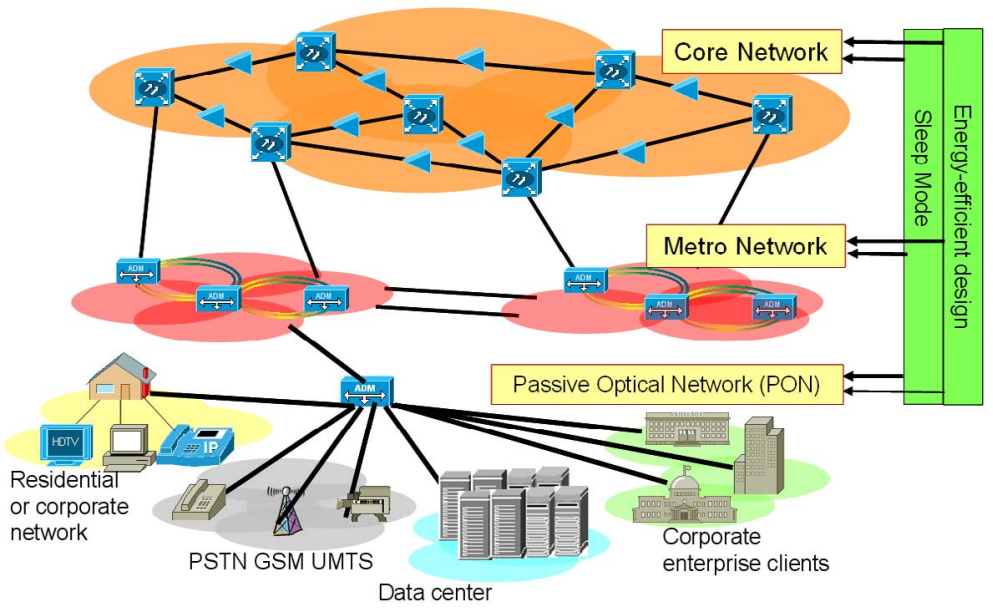

Figure 1. Energy efficiency in optical network segments.

\subsection{Energy Efficiency in Optical Core Networks}

In general, core networks present a multilayer architecture in which electronic routers exploit an underlying static or dynamic optical transport network consisting of optical crossconnects and optical fibre links. Therefore many of the studies available in the literature evaluate the energy consumption of the overall architecture focusing, in particular, on the comparison between an all-electronic, an all-optical and a hybrid architecture. The main results of these studies is that the aggregation of the traffic in high speed (optical) links reduces the energy-per-bit necessary for the transmission. In [13], it is mentioned that the core optical transport (wavelength division multiplexed links) accounts for only a small fraction of the total energy consumed by the Internet, with most energy being consumed by the access network and by the core network electronic routing functions. Optical technologies are therefore seen as a key strategy for a scalable growth of power consumption in the future core networks. Other studies propose to utilize alternative energy sources to power the network devices placed nearby. In this scenario, routing and device virtualization helps different users to exploit the device functionalities. If electronic routers with optical interfaces or all-optical switches are utilized in the core, 
methods based on the possibility of temporarily turning off underutilized interfaces and controlling it through extensions to the GMPLS protocol suite are being proposed [15].

\subsection{Energy Efficiency in Optical Metro Networks}

Currently, the metro and edge segment of the networks consist of mainly electronic devices such as broadband network gateways (BNGs) or Broadband Remote Access Server (BRAS). In addition, provider edge routers represent the gateway to the network core. In recent years, with the foreseen increase in Internet users, proposals for all-optical metro networks started to raise. However the most likely solution is a hybrid architecture, with optical and electronic equipment. Although, optical technology is energy efficient, aggregation of traffic collected from access networks can be performed only in electronic domain. Therefore, a proper optimization of the metro network design to minimize the power consumption of optical and electronic equipment is important [16][17].

\subsection{Energy Efficiency in Optical Access Networks}

In [18] it is shown that ADSLs, PONs, and FTTNs, the customer modems or ONU consume over $65 \%$ of the total power in the access network. Thus, the overall power of the access segment can be drastically reduced by optimizing the energy consumption of the devices at the customer premises. A solution is to exploit the sleep mode (i.e., stand by ) of the equipment when idle, i.e., when not transmitting nor receiving traffic. This solution is currently investigated by the IEEE 802.3az Energy Efficient Ethernet Task Force, to lower energy consumption in copper based Ethernet links using sleep or power save mode [19]. Similar efforts are ongoing within the IEEE 802.3av working group [20] and within the ITU-T. In particular, ITU-T has recently published G.sup45 that considers a number of practical issues to incorporate different ONU power saving techniques in the operators' networks [21].

\section{SLEEP MODE IN OPTICAL ACCESS NETWORKS}

This section addresses the challenge of reducing the energy consumption of passive optical networks (PONs). Most of the studies conducted within both the research community [22][23] and working groups in the standard bodies [20] build upon the idea of allowing PON network elements, specifically the ONUs, to switch to sleep mode for saving energy. The concept of such approach stems from the broadcast and TDMA nature of the PON. Downstream traffic is broadcast to all the ONUs, thus data are received by each ONU, but discarded if not destined to that ONU. This implies wasting significant energy at the ONU side to receive and process the broadcast data. Similarly, if ONU need not transmit, it remains silent while other ONUs are transmitting. Therefore, each ONU can conserve energy by switching to sleep mode when it need not send nor receive traffic.

However, to implement the sleep mode in ONUs, some issues must be solved. In current time division multiple access (TDMA)-Ethernet PON (EPON), ONUs synchronize their local clock to the OLT clock through their clock and data recovery (CDR) circuit that receives the continuous bit stream (data or idle) from the OLT [24]. If an ONU stops receiving downstream transmission, clock synchronization at ONU is lost. Current ONU architecture requires long clock recovery time when the ONU wakes up from the sleep mode. Moreover, the absence of synchronization among different ONUs clocks might cause upstream data collision at the OLT.

In general the energy consumed by each ONU implementing sleep mode during a transmission cycle $T_{C}$ (i.e., the time elapsing between two consecutive upstream data transmission from he same ONU) can be written as:

$$
E_{C}=P_{S} T_{S}+P_{O N}\left(T_{O N}+T_{O H}\right)=\left(P_{O N}-P_{S}\right)\left(T_{O N}+T_{O H}\right)+P_{S} T_{C},
$$

where $P_{O N}$ and $P_{S}$ are the power consumed by the receiving circuit of the ONU during the time in which the ONU is awake $(\mathrm{ON})$ and asleep $(\mathrm{S})$, respectively, $T_{O N}$ is the time for which the ONU is awake (ON), $T_{O H}$ is the time needed by the receiver to synchronize to the OLT clock and regain synchronization with the downstream scheduling (overhead). Eq. (1) shows that the energy consumed by an ONU in a cycle is linearly dependent not only on the time for which the station is awake $(\mathrm{ON})$ but also on the overhead time for ONU synchronization. Moreover, $E_{C}$ is directly proportional to the power consumed during the sleep time $P_{S}$. Thus two dimensions can be exploited to decrease the energy consumed by the ONU: 1) the time, i.e., duration of overheads and periods of activity $(\mathrm{ON})$, and 2 ) the power consumed, especially when the ONU is asleep.

\section{ENERGY EFFICIENT METRO NETWORK DESIGN}

This section addresses the challenge of reducing the energy consumption of metro optical networks. A large number of works studied the problem of minimizing the resources to be allocated in metro networks in order to support the traffic coming/destined from/to the access networks. Minimizing the allocated resources can help to reduce the overall power consumption. However, metro networks are based on an optical layer and electronic layer, that consumes a different amount of power. Therefore, the allocation of resources should account for power drained by both the optical equipments and the electronic equipment. 
In general, the power consumed by a metro ring with $N$ nodes can be written as:

$$
P_{C}=N\left(P_{L}+P_{N}\right)+n_{l}\left(h P_{O}+P_{T R X}\right),
$$

where $n_{l}$ is the number of optical connections required to support the given traffic, $h$ is the average hop-length of the optical connections, and $P_{L} P_{N}, P_{O}, P_{T R X}$ are the power drained by each optical link (e.g., line amplifiers), by each node (i.e., add-drop multiplexers), by an optical interface and optical bypass at a node, and by a transceiver, respectively. Eq. (2) shows that two dimensions can be exploited to reduced the overall power consumption: 1) reducing the power consumption of the equipment and 2) minimizing the number of optical channels to be allocated and their hop length $(h)$. Unfortunately, the number of optical channels grows as $N^{2}$ and may make the second term more relevant. Moreover, when $h$ is small, a large number of optical connections is required, although traffic can be well aggregated. On the contrary, when $h$ is larger, fewer optical connections are required (e.g., traffic can be sent through only one optical connection). The optimal combination of these conflicting trends need to be addressed by optimization algorithms.

\section{CONCLUSIONS}

In this paper a survey of the efforts in both the private and the public sectors toward a more efficient energy utilization in communications networks has been presented. Particular attention has been focused on optical networks due to their potential of higher energy savings. The considered case studies (metro and access optical networks) have shown that through optimal design and control of optical network the energy savings are possible while limiting the impact on the carried traffic.

\section{ACKNOWLEDGEMENTS}

This work has been sponsored by the BONE-project "Building the Future Optical Network in Europe", a Network of Excellence funded by the European Commission through the 7th ICT-Framework Programme.

\section{REFERENCES}

[1] “Climate Change Information Kit”, published by UNOE and UNFCCC, 2002.

[2] Kyoto Protocol Reference Manual, UNFCCC.

[3] "Carbon footprint: An introduction for organisations", Carbon Trust, Aug. 2007.

[4] The climate group, "SMART 2020: Enabling the low carbon economy in the information age", http://www.smart2020.org/ .

[5] Lan Ti and H. R. Thomas, "A review of research on the environmental impact of e-business and ICT", Environment International, vol. 33, no. 6, pp. 841-849, Aug. 2007.

[6] M. Matsumoto et al., "Prospects for an environmentally sustainable ICT society", Eco Design 2005http://unfecc.int/2860.php.

[7] Gartner press release, http://www.gartner.com/it/page.jsp?id=503867, 2007

[8] A. Gladisch; C. Lange; R. Leppla, "Power efficiency of optical versus electronic access networks", ECOC 2008 Symposium on Network Solutions to Reduce the Energy Footprint of ICT.

[9] F. Cucchietti, "Energy efficiency - an enabler for the Next Generation Networks", Bruxelles 2006.

[10] J. Baliga, K. Hinton, R. S. Tucker, "Energy consumption of the Internet", in Proc. COIN 2007.

[11] C. Lange and A. Gladish, "Energy Consumption of Telecommunication Networks: A Network's Operator View", "Energy Footprint of ICT: Forecasts and Network Solutions", in Proc. OFC 2009.

[12] Switching and Energy, (Plenary), Photonics in Switching 2009, Pisa, Italy, Sept. 16-18, 2009.

[13] R. S. Tucker et al., "Evolution of WDM Optical IP Networks: A Cost and Energy Perspective", IEEE/OSA $J L T$, vol. 27, no. 3, pp. 2391-2403, Feb. 1, 2009.

[14] S. Figuerola et al., "Converged optical network infrastructure in support of future Internet and grid services using IaaS to reduce GHG emissions", IEEE/OSA JLT, vol. 27, no. 12, pp. 1941-1946, 2009.

[15] L. Chiaraviglio et al., "Energy-aware backbone networks: A case study", 1st IEEE Workshop on Green Communications GreenComm, IEEE ICC 2009.

[16] I. Cerutti et al., "Designing power-efficient WDM ring networks", Gridnets 2009 [INVITED].

[17] E. Yetginer and G. Rouskas, "Power efficient traffic grooming in optical WDM networks", Globecom 2009.

[18] J. Baliga et al., "Energy consumption in optical IP networks", IEEE/OSA JLT, vol. 27, no. 13, Jul. 1, 2009.

[19] http://www.ieee802.org/3/az/index.html

[20] J. Mandin, "EPON power saving via sleep mode," IEEE 802.3av Meeting, Sep. 2008.

[21] “GPON Power Conservation”, ITU-T G-series Rec. - Supplement 45 (G.sup45), ITU-T, 05/2009.

[22] T. Smith, R. S. Tucker, K. Hinton, and A. V. Tran, "Implications of sleep mode on activation and ranging protocols in PONs," IEEE LEOS 2008.

[23] S.-W. Wong, et al., "Sleep mode for energy saving PONs: Advantages and drawbacks", 2nd IEEE Workshop on Green Communications GreenComm, Globecom 2009.

[24] C. Lam (Ed.), "Passive Optical Networks: Principles and Practice”, Academic Press-Elsevier, 2007. 